\title{
Coeliac disease and invasive pneumococcal disease: a population-based cohort study
}

\author{
A. RÖCKERT TJERNBERG ${ }^{1,2} *$ J. BONNEDAHL ${ }^{3,4}$, M. INGHAMMAR ${ }^{5}$, \\ A. EGESTEN ${ }^{6}$, G. KAHLMETER ${ }^{7}$, P. NAUCLÉR ${ }^{8,9}$, \\ B. HENRIQUES-NORMARK ${ }^{10,11}$ AND J. F. LUDVIGSSON ${ }^{12,13,14}$
}

\begin{abstract}
${ }^{1}$ Department of Paediatrics, Kalmar County Hospital, Kalmar, Sweden; ${ }^{2}$ School of Health and Medical Sciences, Örebro University, Sweden; ${ }^{3}$ Department of Infectious Diseases, Kalmar County Hospital, Kalmar, Sweden; ${ }^{4}$ Zoonotic Ecology and Epidemiology, Faculty of Health and Life Sciences, Linnaeus University, Sweden; ${ }^{5}$ Lund University, Department of Clinical Sciences Lund, Section for Infection Medicine, Lund, Sweden;

${ }^{6}$ Respiratory Medicine and Allergology, Department of Clinical sciences, Lund University, Lund, Sweden;

${ }^{7}$ Department of Clinical Microbiology, Central Hospital, Växjö, Sweden; ${ }^{8}$ Department of Infectious Diseases, Karolinska University Hospital, Stockholm, Sweden; ${ }^{9}$ Department of Medicine, Solna, Infectious Diseases Unit, Karolinska Institutet, Stockholm, Sweden; ${ }^{10}$ Department of Clinical Microbiology, Karolinska University Hospital, Stockholm, Sweden; ${ }^{11}$ Department of Microbiology, Tumor and Cell Biology, Karolinska Institutet, Stockholm, Sweden; ${ }^{12}$ Department of Medical Epidemiology and Biostatistics, Karolinska Institutet, Stockholm, Sweden; ${ }^{13}$ Department of Paediatrics, Örebro University Hospital, Sweden; ${ }^{14}$ Division of Epidemiology and Public Health, School of Medicine, University of Nottingham, City Hospital, Nottingham, UK
\end{abstract}

Received 16 August 2016; Final revision 8 December 2016; Accepted 9 December 2016; first published online 23 January 2017

\section{SUMMARY}

Severe infections are recognized complications of coeliac disease (CD). In the present study we aimed to examine whether individuals with $\mathrm{CD}$ are at increased risk of invasive pneumococcal disease (IPD). To do so, we performed a population-based cohort study including 29012 individuals with biopsy-proven CD identified through biopsy reports from all pathology departments in Sweden. Each individual with CD was matched with up to five controls $(n=144$ 257). IPD events were identified through regional and national microbiological databases, including the National Surveillance System for Infectious Diseases. We used Cox regression analyses to estimate hazard ratios (HRs) for diagnosed IPD. A total of 207 individuals had a record of IPD whereas 45/29 012 had CD $(0 \cdot 15 \%)$ and 162/144 257 were controls $(0 \cdot 11 \%)$. This corresponded to a $46 \%$ increased risk for IPD [HR 1·46, 95\% confidence interval (CI) 1·05-2·03]. The risk estimate was similar after adjustment for socioeconomic status, educational level and comorbidities, but then failed to attain statistical significance (adjusted HR 1.40, 95\% CI 0.991.97). Nonetheless, our study shows a trend towards an increased risk for IPD in CD patients. The findings support results seen in earlier research and taking that into consideration individuals with $\mathrm{CD}$ may be considered for pneumococcal vaccination.

Key words: Coeliac disease, pneumococcal infection, pneumococci, septicaemia.

\footnotetext{
* Author for correspondence: Dr A. Röckert Tjernberg, Department of Paediatrics, Kalmar County Hospital, S-391 85 Kalmar, Sweden.

(Email: annart@ltkalmar.se)
}

\section{INTRODUCTION}

Patients with coeliac disease (CD) have been suggested to be more susceptible to infectious diseases [1]. Hence individuals with $\mathrm{CD}$ are considered to be at increased 
risk for influenza requiring hospital care [2] and tuberculosis [3]. In addition, CD has been linked to sepsis [4], with a particularly high risk for sepsis caused by pneumococci [hazard ratio (HR) 3.9, 95\% (CI) confidence interval 2.2-7.0] [5].

Several factors may contribute to this susceptibility including an increased intestinal permeability [6] and hyposplenism (hypofunction of the spleen with or without atrophy) in CD [7].

Streptococcus pneumoniae (pneumococcus) is a Gram-positive encapsulated bacterium. This widespread pathogen is one of the most common causes of bacterial respiratory tract infections [8] including otitis media, especially in children [9], but pneumococci are also a common cause of life-threatening disorders such as sepsis and meningitis $[8,10]$.

Considering that several studies have demonstrated an increased risk of sepsis in CD [4,5] and the fact that $\mathrm{CD}$ has been linked to hyposplenism, it is surprising that so far only one study has specifically examined the risk for invasive pneumococcal disease (IPD) in CD [11]. In the current Swedish study we examined the risk of IPD in more than 29000 individuals with biopsy-verified CD compared to the general population. We hypothesized that individuals with $\mathrm{CD}$ would be at increased risk of IPD.

\section{METHODS}

Nationwide data from biopsy reports were used to identify individuals with $\mathrm{CD}$. In a population-based cohort study these were matched to subjects without $\mathrm{CD}$ (controls) from the general population and thereafter linked with healthcare databases containing information on IPD. Linkage of all the databases was performed by the National Board for Health and Welfare to keep the data anonymous.

\section{Study participants (coeliac individuals and controls)}

Data on small intestinal biopsies were collected from all Swedish pathology departments $(n=28)$ during 2006-2008. The biopsies had been performed in 1969-2008, with a majority of patients undergoing biopsy in 1990 or later (Table 1). CD was defined as having villous atrophy (VA), which is equivalent to Marsh histopathology stage III. Local IT personnel at each pathology department collected the biopsy reports and delivered data on personal identity number, date of biopsy, topography and morphology according to the Swedish SnoMed classification (see
Table 1. Characteristics of study participants

\begin{tabular}{lll}
\hline \hline & $\begin{array}{l}\text { Controls, } \\
n(\%)\end{array}$ & $\begin{array}{l}\text { Coeliac disease, } \\
n(\%)\end{array}$ \\
Characteristics & $144257(100)$ & $29012(100)$ \\
$\begin{array}{l}\text { Total } \\
\text { Sex }\end{array} \quad$ & \\
$\quad$ Male & $54835(38)$ & $11040(38 \cdot 1)$ \\
Female & $89422(62)$ & $17972(61 \cdot 9)$ \\
Age, years* & & \\
$0-19$ & $58818(40 \cdot 8)$ & $11796(40 \cdot 7)$ \\
$20-39$ & $26368(18 \cdot 3)$ & $5306(18 \cdot 3)$ \\
$40-59$ & $32205(22 \cdot 3)$ & $6457(22 \cdot 3)$ \\
$\geqslant 60$ & $26866(18 \cdot 6)$ & $5453(18 \cdot 8)$ \\
Calendar year* & & \\
1969-1989 & $20142(14)$ & $4029(13 \cdot 9)$ \\
1990-1999 & $59864(41 \cdot 5)$ & $12055(41 \cdot 6)$ \\
2000-2009 & $64251(44 \cdot 5)$ & $12928(44 \cdot 6)$ \\
Country of birth & & \\
$\quad$ Nordic & $136017(94 \cdot 3)$ & $28056(96 \cdot 7)$ \\
$\quad$ Not Nordic & $8240(5 \cdot 7)$ & $956(3 \cdot 3)$ \\
Comorbidity & & \\
$\quad$ Diabetes $\dagger$ & $595(0 \cdot 4)$ & $954(3 \cdot 3)$ \\
Liver disease & $4880(3 \cdot 4)$ & $1524(5 \cdot 3)$ \\
End-stage renal disease & $235(0 \cdot 2)$ & $135(0 \cdot 5)$ \\
Asthma & $6363(4 \cdot 4)$ & $1935(6 \cdot 7)$ \\
Ischaemic heart disease & $8322(5 \cdot 8)$ & $2141(7 \cdot 4)$ \\
\hline \hline
\end{tabular}

* At time of coeliac disease diagnosis.

$\dagger$ Diagnosed at age $\leqslant 30$ years.

Supplementary Table S1) to the research group. A positive $\mathrm{CD}$ serology was not required for a $\mathrm{CD}$ diagnosis; however, a previous validation of a random subset of individuals with biopsy-proven CD (VA) found that $88 \%$ of patients were serologically positive at the time of biopsy [12]. After data irregularities and duplicates were removed we identified 29096 individuals with VA of whom 29012 had a follow-up extending to 1987 or beyond and hence were included in the study.

The Swedish government agency, Statistics Sweden, thereafter matched each individual with up to five randomly selected controls from the Swedish Total Population Register (1 44257 of these had a follow-up to 1987 or beyond). Matching criteria included age, sex, calendar year, county and the controls were not to have any prior record of small intestinal biopsy. Details on this data collection have been published elsewhere [12].

\section{Demographic data}

We obtained data on socioeconomic status (occupational data), educational level (a detailed description 
has been published elsewhere [13]) and country of birth from Statistics Sweden (the Swedish government agency responsible for producing official statistics). Information from the different databases were linked using the Swedish personal identity number [14]. We did not have access to data on smoking or alcohol consumption.

\section{Data on pneumococcal infections}

We defined IPD as growth of Streptococcus pneumoniae in a culture from a normally sterile site [i.e. blood, cerebrospinal fluid (CSF), pleural effusion, pericardial fluid, or synovial fluid]. Outcome data were obtained from two sources (see below), and linked to our biopsy data through the personal identity number [14] a unique number assigned to all Swedish residents.

(i) Nationwide data from the Public Health Agency of Sweden. In Sweden, IPD has been a notifiable disease since 1 July 2004 and data on IPD are consecutively registered at the Public Health Agency of Sweden (formerly Swedish Institute for Infectious Disease Control). The reporting system includes both active reporting from clinicians as well as automatic reporting from all Sweden's microbiological laboratories resulting in an almost 100\% coverage (from 2005 and onwards).

(ii) Data from Southern Sweden were obtained from computerized databases from the seven microbiological laboratories covering the area: Växjö (representing Kronoberg region) 1987-2004, Kalmar 1990-2004, Malmö (Skane region) 1990-2004, Kristianstad (Skane) 1991-2004, Lund (Skane) 1991-2004, Karlskrona (Blekinge region) 1994-2004 and Halmstad (Halland region) 1995-2004.

\section{Statistics}

Cox regression was used to estimate HRs for IPD in coeliac patients compared to controls. Proportional hazards assumption was verified using log-minus-log curves. Follow-up started with date of first biopsy (and equal date in matched controls). Follow-up ended at IPD (positive culture), death, emigration or end of study (31 December 2009), whichever occurred first. HRs were adjusted for level of education, socioeconomic status, country of birth and in a separate analysis also for several possible confounding comorbidities. Diabetes has a strong association with $\mathrm{CD}$ [15] and is also linked to IPD [16], while we considered diabetes to be a potential major confounder. We also adjusted for liver disease, end-stage renal disease, asthma and ischaemic heart disease (defined according to relevant ICD codes), conditions which are all linked with CD [17-20] as well as with IPD [16, 21, 22]. Since each individual with $C D$ was compared to his/her matched controls, matching variables were automatically considered (in this regard our analysis resembled a conditional logistic regression). Follow-up specific HRs for IPD $(<1$ year, 14.99 years and $\geqslant 5$ years) were calculated. In addition we performed a priori-defined sub-analyses stratifying for age, sex and calendar period (1969-1989, 1990 1999, 2000-2009) at CD diagnosis. The statistical significance of difference in risk estimates between strata was tested with formal interaction tests. In a final analysis we also restricted our study population to include only individuals diagnosed with CD (and IPD) 1994 or later (the year when the IPD database from southern Sweden became complete) and also to individuals diagnosed 2004 and beyond (the year when IPD became a notifiable disease).

We used SPSS v. 22 (IBM Corp., USA) for all analyses. HRs with a 95\% CI not including 1 and $P<0.05$ were considered statistically significant.

\section{Ethical approval}

This study was approved by the Stockholm Ethics Review Board (2006/633-31/4). Since data were strictly register-based no individual consent was required.

\section{RESULTS}

The median age at IPD diagnosis was 62.3 years in the CD group and 68.7 years in the control group. The mean follow-up time (i.e. duration from $\mathrm{CD}$ diagnosis to IPD) was $10 \cdot 5$ years in individuals with $\mathrm{CD}$ and $11 \cdot 6$ years in the control group. Nearly all the IPD cases had bacterial growth in blood and only a few in CSF and other sites. Additional details of the study participants are presented in Table 1 .

Out of 173269 study participants, 207 had a record of IPD. The proportion of patients with IPD was higher in the CD group (45/29 012, $0 \cdot 15 \%$ ) than in controls $(162 / 144257,0 \cdot 11 \%)$. Hence the HR for encountering IPD was 1.46 in the CD group compared to the general population $(95 \%$ CI $1 \cdot 05-2 \cdot 03)$. 
Table 2. Risk of IPD based on follow-up time in individuals with coeliac disease

\begin{tabular}{lllllr}
\hline \hline Follow-up & Observed events & Expected events & HR $(95 \%$ CI $)$ & $P$ value & Attributable percentage* \\
\hline All & 45 & 31 & $1 \cdot 46(1 \cdot 05-2 \cdot 03)$ & $0 \cdot 025$ & 32 \\
Year $<1$ & 1 & 2 & $0 \cdot 58(0 \cdot 08-4 \cdot 52)$ & $0 \cdot 61$ & -72 \\
Year $1-4 \cdot 99$ & 10 & 4 & $2 \cdot 25(1 \cdot 08-4 \cdot 70)$ & $0 \cdot 03$ & 56 \\
Year $\geqslant 5$ & 34 & 25 & $1 \cdot 38(0 \cdot 94-2 \cdot 01)$ & $0 \cdot 10$ & 27 \\
\hline \hline
\end{tabular}

IPD, Invasive pneumococcal disease; HR, hazard ratio; CI, confidence interval.

Reference is general population comparator cohort.

* Calculated as $[1-(1 / \mathrm{HR})] \times 100$.

Table 3. Risk of IPD in relation to characteristics of patients with coeliac disease

\begin{tabular}{|c|c|c|c|c|c|}
\hline Subgroup & Observed events & Expected events & HR $(95 \% \mathrm{CI})$ & $P$ value & Attributable percentage* \\
\hline \multicolumn{6}{|l|}{ Sex } \\
\hline Males & 8 & 11 & $0.70(0.33-1 \cdot 47)$ & 0.35 & -43 \\
\hline Females & 37 & 20 & $1.90(1 \cdot 30-2 \cdot 76)$ & $0 \cdot 001$ & 47 \\
\hline \multicolumn{6}{|l|}{ Age, years $\dagger$} \\
\hline$<20$ & 5 & 2 & $2 \cdot 24(0 \cdot 79-6 \cdot 37)$ & $0 \cdot 13$ & 55 \\
\hline $20-39$ & 6 & 5 & $1 \cdot 18(0 \cdot 49-2 \cdot 85)$ & $0 \cdot 71$ & 15 \\
\hline $40-59$ & 21 & 12 & $1 \cdot 81(1 \cdot 11-2 \cdot 96)$ & $0 \cdot 018$ & 45 \\
\hline$\geqslant 60$ & 13 & 12 & $1.08(0.59-1.98)$ & $0 \cdot 81$ & 7 \\
\hline \multicolumn{6}{|c|}{ Calendar period $\dagger$} \\
\hline 1969-1989 & 8 & 6 & $1 \cdot 31(0 \cdot 60-2 \cdot 89)$ & $0 \cdot 50$ & 24 \\
\hline 1990-1999 & 22 & 14 & $1.55(0.96-2.50)$ & 0.07 & 36 \\
\hline 2000-2008 & 15 & 11 & $1.42(0.81-2.51)$ & $0 \cdot 22$ & 30 \\
\hline
\end{tabular}

IPD, Invasive pneumococcal disease; HR, hazard ratio; CI, confidence interval.

Reference is general comparator cohort.

* Calculated as $[1-(1 / \mathrm{HR})] \times 100$.

$\uparrow$ At coeliac disease diagnosis and corresponding age in controls.

Adjusting for education, economy and country of birth did not influence the risk estimate [adjusted hazard ratio (aHR) 1·48, 95\% CI 1·06-2.07]. In a separate analysis where we also added type 1 diabetes, chronic liver disease, end-stage renal disease, asthma and ischaemic heart disease as covariates in our statistical model, the HR was 1.40 (95\% CI 0.99-1.97)

To minimize the risk of missing IPD cases, in a separate analysis we also restricted the study population to individuals diagnosed with CD in 1994 or later (the year when the regional microbiological database became complete). Restricting study participants to the last two decades did, however, not influence our risk estimate for IPD more than marginally (aHR $1 \cdot 58,95 \%$ CI $1 \cdot 05-2 \cdot 37$ ). We had also intended to examine the risk of IPD in coeliac patients diagnosed during 2004-2008 but due to lack of positive IPD cases we were unable to calculate any HRs.

The highest risk for IPD was seen $1-4.99$ years after CD diagnosis (HR 2.25, 95\% CI 1.08-4.70), with lower HRs both at 1 year and $\geqslant 5$ years follow-up (Table 2). The risks according to follow-up were not substantially influenced by comorbidities. The increased risk for IPD was mainly seen in women (HR 1.90, 95\% CI 1.30-2.76) and a formal test for interaction revealed a statistically significant difference between sexes $(P=0 \cdot 017)$. We found similar risk estimates in the different age groups ( $P$ for interaction $=0 \cdot 2$ ). Further data on risk of IPD in relation to characteristics (sex, age, calendar period) at CD diagnosis are summarized in Table 3.

\section{DISCUSSION}

In this large population-based cohort study we found a $46 \%$ increased risk of IPD in patients with CD (HR $1 \cdot 46,95 \%$ CI $1 \cdot 05-2 \cdot 03)$. The excess risk of IPD seen in our study is consistent with earlier reports of a positive association between $\mathrm{CD}$ and pneumococcal disease (in a British study, Thomas et al. found a 
twofold risk increase for IPD in CD [11] and in 2008 our group reported an almost fourfold increased risk for pneumococcal sepsis [5]). The slightly lower risk estimates in the current study might be due to our access to outpatient data while the British study as well as the earlier Swedish study were mainly limited to inpatients, patients potentially suffering from a more severe $\mathrm{CD}$ than the average patient. In addition, biopsy was mandatory for the diagnosis of $\mathrm{CD}$ in our study. Therefore, misclassification is minimized, since the diagnoses are based on biopsy report data. Likewise, our IPD diagnoses were based on positive cultures and not ICD codes, probably reducing the number of false positives. Furthermore, in the present study we have been able to adjust for diabetes as well as several other potentially confounding comorbidities, which was not done in the British study [11].

During the last 10 years the incidence of IPD in Sweden has varied between 12 and 19/100 000 personyears and most cases are aged $>60$ years [23]. IPD is a severe and potentially life-threatening condition. Taking that into account, we consider the almost $50 \%$ increased risk of IPD in individuals with CD in our study important. The attributable fraction of $\mathrm{CD}$ was as high as $32 \%$ (Table 2). Our results indicate that patients with $\mathrm{CD}$ are a risk group for pneumococcal disease and therefore CD patients may be considered for pneumococcal vaccination also in countries, which unlike the UK [24], do not yet recommend such vaccinations. However, due to herd immunity and serotype replacement, caused by pneumococcal vaccination in the childhood immunization programme introduced in Sweden in 2009, the potential serotype coverage of these vaccines in $\mathrm{CD}$ patients need to be addressed in future studies [25].

One of the most frequently suggested mechanisms explaining the elevated risk for infectious diseases in $\mathrm{CD}$ is CD-associated hyposplenism. Many researchers consider $\mathrm{CD}$ to be a major cause of splenic hypofunction or atrophy. However, the clinical significance of CD-associated hyposplenism is still not fully understood, but there is evidence suggesting that splenic atrophy can cause a deficiency of IgM memory B cells, which are crucial for the immunity against encapsulated bacteria [26, 27]. The prevalence of hyposplenism in CD varies between $19 \%$ and $80 \%$ and is highest in individuals with complicated CD or additional autoimmune diseases [28] and it is mainly seen in adults [29]. Studies are contradictory regarding whether this dysfunction is reversible or not [30, 31]. It is, however, believed by some authors that the hypofunction is reversible after introduction of a gluten-free diet but an atrophy of the spleen might remain [26]. Considering this possibility of improvement of spleen function we also calculated risks for IPD based on follow-up time (Table 2). However this sub-analysis reduced the study power and the confidence intervals for the IPD risk in our three $a$ priori-defined follow-up periods overlapped and therefore we cannot state that the IPD excess risk really decreases over time.

The major strength of this study is its high validity. Both the CD and the IPD diagnoses are highly reliable. The CD diagnoses were based on biopsy reports showing VA, and small-intestinal biopsy was the gold standard for diagnosing CD during the whole study period. The histopathology examinations were carried out by a large number of pathologists. It was not possible to carry out an agreement rate for CD diagnosis between all these pathologists, but when representatives from the different pathology departments carried out a blinded test, $90 \%$ (95\% CI 87-94) of the biopsies with VA (according to the national steering group of small intestinal pathology) were correctly classified [12]. Likewise, the IPD diagnoses are based on positive cultures and active as well as automatic reporting, minimizing the risk for misclassification. However, we cannot rule out that IPD cases have been missed (false negatives), especially since we only had access to regional data from the first part of the study period. Despite this, our study was large and had a long follow-up time during which we identified more than 200 IPD cases. This was sufficient to demonstrate a positive association with $\mathrm{CD}$, but generally insufficient to confirm an IPD excess risk in sub-analyses. Another limitation is that we lacked information on actual hyposplenism. Splenic size and function are not routinely examined in patients with $\mathrm{CD}$ and therefore hyposplenism is not recorded in Swedish national registers. In addition, we did not have data on vaccination status. However, pneumococcal immunization was introduced in the Swedish national vaccine programme as late as 2009 [32] and has been recommended for elderly people (>65 years), since 1994 [33] but not routinely to individuals with $\mathrm{CD}$ so vaccinations are therefore unlikely to have affected our relative risks more than marginally. Smoking is considered to be a risk factor for IPD [34], but due to lack of smoking data we were unable to adjust for smoking. Given its neutral [35] or negative [36] association with $\mathrm{CD}$ smoking should not explain the positive association between CD and IPD. Likewise, we 
lack data on alcohol abuse; however, we have been able to adjust our risk estimate for socioeconomic status which might serve as a proxy for both these variables. Furthermore, other chronic illnesses than CD, such as diabetes, are known to influence the incidence of IPD [16, 22, 37]. Diabetes is common in individuals with $\mathrm{CD}[15]$ and since the risk for splenic hypofunction is increased in individuals with $\mathrm{CD}$ who also have additional autoimmune diseases [28] we chose to adjust for diabetes (as well as liver disease, end-stage renal disease, asthma and ischaemic heart disease, which are also linked to $C D$ ) in our analyses. This did not influence our risk estimates substantially; however, after adjustments the risk estimates just failed to reach statistical significance and diabetes is certainly an important risk factor for IPD.

In conclusion, this study found a moderately increased risk for IPD in individuals with $C D$. The positive association between $\mathrm{CD}$ and pneumococcal disease supports earlier findings in this field [11] and is consistent with current UK guidelines on CD [24]. Taken together this suggests that preventive pneumococcal vaccination may be considered in individuals with CD.

\section{SUPPLEMENTARY MATERIAL}

For supplementary material accompanying this paper visit https://doi.org/10.1017/S0950268816003204.

\section{ACKNOWLEDGEMENTS}

We thank the Public Health Agency of Sweden for support. This work was supported by Kalmar County Council (A.R.T., J.B.) and grants from the Swedish Society of Medicine, the Swedish Research Council and the Swedish Coeliac Society (J.F.L.). B.H.N. was supported by grants from the Swedish Research Council and by ALF grant from the Stockholm County Council.

\section{DECLARATION OF INTEREST}

None.

\section{REFERENCES}

1. Walters JR, Bamford KB, Ghosh S. Coeliac disease and the risk of infections. Gut 2008; 57: 1034-1035.

2. Marild K, Fredlund H, Ludvigsson JF. Increased risk of hospital admission for influenza in patients with celiac disease: a nationwide cohort study in Sweden.
American Journal of Gastroenterology 2010; 105: 2465-2473.

3. Ludvigsson JF, et al. Risk of tuberculosis in a large sample of patients with coeliac disease - a nationwide cohort study. Alimentary Pharmacology \& Therapeutics 2011; 33: 689-696.

4. Peters U, et al. Causes of death in patients with celiac disease in a population-based Swedish cohort. Archives of Internal Medicine 2003; 163: 1566-1572.

5. Ludvigsson JF, et al. Coeliac disease and risk of sepsis. Gut 2008; 57: 1074-1080.

6. Bjarnason I, et al. Intestinal permeability in patients with coeliac disease and dermatitis herpetiformis. Gut 1985; 26: 1214-1219.

7. Muller AF, Toghill PJ. Hyposplenism in gastrointestinal disease. Gut 1995; 36: 165-167.

8. O'Brien KL, et al. Burden of disease caused by Streptococcus pneumoniae in children younger than 5 years: global estimates. Lancet 2009; 374: 893-902.

9. Eskola J, et al. Efficacy of a pneumococcal conjugate vaccine against acute otitis media. New England Journal of Medicine 2001; 344: 403-409.

10. Bogaert D, De Groot R, Hermans PW. Streptococcus pneumoniae colonisation: the key to pneumococcal disease. Lancet Infectious Diseases 2004; 4: 144-154.

11. Thomas HJ, et al. Pneumococcal infection in patients with coeliac disease. European Journal of Gastroenterology \& Hepatology 2008; 20: 624-628.

12. Ludvigsson JF, et al. Validation study of villous atrophy and small intestinal inflammation in Swedish biopsy registers. BMC Gastroenterology 2009; 9: 19.

13. Olen $\mathbf{O}$, et al. Socioeconomic position and education in patients with coeliac disease. Digestive and Liver Disease 2012; 44: 471-476.

14. Ludvigsson JF, et al. The Swedish personal identity number: possibilities and pitfalls in healthcare and medical research. European Journal of Epidemiology 2009; 24: 659-667.

15. Elfstrom P, Sundstrom J, Ludvigsson JF. Systematic review with meta-analysis: associations between coeliac disease and type 1 diabetes. Alimentary Pharmacology \& Therapeutics 2014; 40: 1123-1132.

16. Torres A, et al. Which individuals are at increased risk of pneumococcal disease and why? Impact of COPD, asthma, smoking, diabetes, and/or chronic heart disease on community-acquired pneumonia and invasive pneumococcal disease. Thorax 2015; 70: 984-989.

17. Reilly NR, et al. Increased risk of non-alcoholic fatty liver disease after diagnosis of celiac disease. Journal of Hepatology 2015; 62: 1405-1411.

18. Welander A, et al. Increased risk of end-stage renal disease in individuals with coeliac disease. Gut 2012; 61: 64-68.

19. Ludvigsson JF, et al. Celiac disease confers a 1·6-fold increased risk of asthma: a nationwide population-based cohort study. Journal of Allergy and Clinical Immunology 2011; 127: 1071-1073.

20. Ludvigsson JF, et al. Nationwide cohort study of risk of ischemic heart disease in patients with celiac disease. Circulation 2011; 123: 483-490. 
21. van Hoek AJ, et al. The effect of underlying clinical conditions on the risk of developing invasive pneumococcal disease in England. Journal of Infection 2012; 65: 17-24.

22. Inghammar M, et al. Invasive pneumococcal disease in patients with an underlying pulmonary disorder. Clinical Microbiology and Infection 2013; 19: 11481154.

23. Public Health Agency. Invasive pneumococcal infections.

24. Ludvigsson JF, et al. Diagnosis and management of adult coeliac disease: guidelines from the British Society of Gastroenterology. Gut 2014; 63: 1210-1228.

25. Galanis I, et al. Effects of PCV7 and PCV13 on invasive pneumococcal disease and carriage in Stockholm, Sweden. European Respiratory Journal 2016; 47: 1208-1218.

26. Di Sabatino A, Carsetti R, Corazza GR. Post-splenectomy and hyposplenic states. Lancet 2011; 378: 86-97.

27. Kruetzmann S, et al. Human immunoglobulin M memory B cells controlling Streptococcus pneumoniae infections are generated in the spleen. Journal of Experimental Medicine 2003; 197: 939-945.

28. Di Sabatino A, et al. Splenic hypofunction and the spectrum of autoimmune and malignant complications in celiac disease. Clinical Gastroenterology and Hepatology 2006; 4: 179-186.
29. Corazza GR, et al. Splenic function in childhood coeliac disease. Gut 1982; 23: 415-416.

30. Corazza GR, et al. Effect of gluten-free diet on splenic hypofunction of adult coeliac disease. Gut 1983; 24: 228-230.

31. Trewby PN, et al. Splenic atrophy in adult coeliac disease: is it reversible? Gut 1981; 22: 628-632.

32. Center for Disease Control (Public Health Agency). Surveillance of the immunization program against pneumococci in children.

33. Ortqvist A, Hedlund J, Kalin M. The elderly should be vaccinated against pneumococci [in Swedish]. Lakartidningen 1999; 96: 1305-1308.

34. Cruickshank HC, Jefferies JM, Clarke SC. Lifestyle risk factors for invasive pneumococcal disease: a systematic review. BMJ Open 2014; 4: e005224.

35. Ludvigsson JF, Nordenvall C, Jarvholm B. Smoking, use of moist snuff and risk of celiac disease: a prospective study. BMC Gastroenterology 2014; 14: 120.

36. Snook JA, et al. Smoking benefits celiac sprue and pouchitis: implications for nicotine therapy? Gastroenterology 1997; 112: 1048-1050.

37. Kyaw MH, et al. The influence of chronic illnesses on the incidence of invasive pneumococcal disease in adults. Journal of Infectious Diseases 2005; 192: 377-386. 